\title{
Steering, optic flow, and the respective importance of depth and retinal motion distribution
}

\author{
Simon K Rushton \\ Department of Psychology, University of Edinburgh, 7 George Square, Edinburgh EH8 9JZ, Scotland \\ Julie M Harris \\ Department of Psychology, Ridley Building, Claremont Place, University of Newcastle, \\ Newcastle-upon-Tyne NE1 7RU, UK \\ John P Wann \\ Department of Psychology, University of Reading, 3 Earley Gate, Reading RG6 6AL, UK \\ Paper submitted in conjunction with the Applied Vision Association Conference/Workshop on Depth \\ Perception, Guildford, Surrey, UK, 4 September 1997; revised version received 19 February 1999
}

\begin{abstract}
Movement through an environment produces an optical spatiotemporal pattern, known as a flow field. When visually guiding movement using a flow field, do humans make use of information about the distance of constituent elements? Employing a novel active steering task, we examined the use of depth (height-in-scene and disparity) and the role of the retinal motion distribution in the perceptual control of heading from flow. We found that retinal motion distribution, rather than depth order, has the primary role in determining the accuracy of steering.
\end{abstract}

\section{Introduction}

During self-motion, instantaneous heading and path are mathematically specified in the retinal flow field (Gibson 1950). In principle, knowledge of the depth of objects in a flow field (the depth of objects could be obtained from a variety of sources, such as perspective, texture gradients, and binocular disparity) can simplify the determination of heading. In particular, it has been argued that, during a pursuit eye movement, depth may assume a critical role in the perception of heading (van den Berg and Brenner 1994a). If an observer makes an eye movement during locomotion, then the retinal flow field is complicated (Regan and Beverley 1982). Van den Berg (1992) hypothesised that the specific role of depth is to identify near and distant objects: the retinal motion of near objects being primarily determined by observer movement, the motion of far objects being primarily determined by eye movement. Thus, if the depth of respective objects in the scene is known, a perceptual system can utilise the movement of distant objects to obtain the rotational component (the component due to eye or head movements). Then, rotation could be subtracted from the overall flow leaving a simple translational component (van den Berg and Brenner 1994a).

Consensus had been building for the use of depth in the perceptual judgment of heading from flow (Cutting 1996; Palmisano 1996; Warren 1995). First, there is a performance difference associated with locomotion over a ground plane compared to that through a cloud (van den Berg 1992). It was hypothesised that this occurs because a ground plane provides depth-order information - the height of an element in the scene is related to its distance from the observer (Sedgwick 1986). Second, it has also been reported that, in the presence of noise, heading judgment performance is better with stereo depth than without it (van den Berg and Brenner 1994b) and that distant points appear to be necessary for accurate heading judgments (van den Berg and Brenner 1994a). Third, the incorporation of depth order in recent computational neural models of human

- Current address to which all correspondence and requests for reprints should be sent: Cambridge Basic Research, Nissan Research \& Development Inc., 4 Cambridge Center, Cambridge, MA 02142, USA; e-mail: simon@cbr.com. 
heading perception (Warren 1995) is bolstered by the finding that neurons in brain area MSTd (a candidate for involvement in the perception of locomotion) are sensitive to binocular disparity (Roy and Wurtz 1990). However, some recent work (Ehrlich et al 1998; Stone and Perrone 1997) is beginning to call into question this consensus.

The role of depth in steering (the perceptual control of locomotor heading) has not been studied experimentally. It has been suggested that perceptual control of action may involve different systems from those implicated in perceptual judgments (Gibson 1979; Goodale and Milner 1992; Milner and Goodale 1996; Warren 1995). Thus it is not currently clear that the results of heading judgment experiments can tell us about the control of heading. Additionally, even if the same systems are involved, it can be argued that perceptual judgment tasks quantify only the ability of an observer to use a tightly constrained set of information sources and strategies. In natural locomotor control there may be additional strategies and information sources that the observer can exploit. This point is discussed in section 2 below.

In this paper we describe a replication of the ground versus cloud and presence versus absence of disparity manipulations, but using a steering task, rather than heading judgments. The aim was to reveal a possible role for depth in steering. A difference in outcome between the judgment and steering results would also be suggestive of the use of different systems, strategies, or information. Our results for control of heading were very similar to those found previously for perceptual judgments (van den Berg and Brenner 1994b). In summary, we found that depth does not improve the control of locomotor heading.

Before the experiments are described, we start by reappraising the depth and heading judgment results. In doing so we discuss some additional control conditions, which we added to our experiment to take account of the potential shortcomings of the previous work.

\subsection{Ground plane versus cloud-an issue of depth or distribution?}

It has been noted that there is a performance difference associated with locomotion over a ground plane as compared to that through a cloud (van den Berg 1992). It was suggested that the performance difference followed from a difference in depth-order informationin the ground-plane condition, optical height specifies depth order (eg points near the horizon are always far away). However, a performance difference can be accounted for without recourse to a depth-order argument.

There is a marked difference in the spatial distribution of dots (and motion vectors) in the upper and lower portions of the visual array resulting from a cloud compared to a ground-plane display (see figure 1). In previous studies of heading judgment, a fixed number of dots were distributed over a plane or through a cloud. For a ground plane, all the dots fall in the lower visual field with the highest density just below the point of fixation. For a cloud, the distribution is sparser and relatively uniform. If the mean retinal dot speed is compared, then it will invariably differ in the two conditions unless it is explicitly controlled for.

Perception of heading must be constrained by the sensitivity to the local motion vectors that describe the flow field (Crowell and Banks 1993, 1996). Sensitivity to motion varies with retinal position and speed (see, for example, Albright 1984; McKee and Nakayama 1984); therefore it is likely that some differences in performance between a ground plane and a cloud can be explained by the difference in low-level motion distribution parameters (locus and mean speed).

A further, more ecologically motivated, difference between a cloud and a ground plane stems from the suggestion that surfaces such as the ground plane may have a special status in human vision (Gibson 1979; He and Nakayama 1994). 

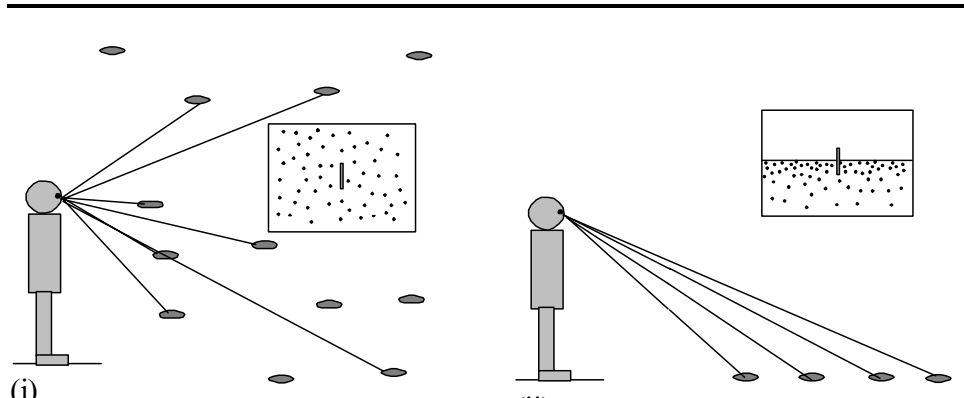

(ii)
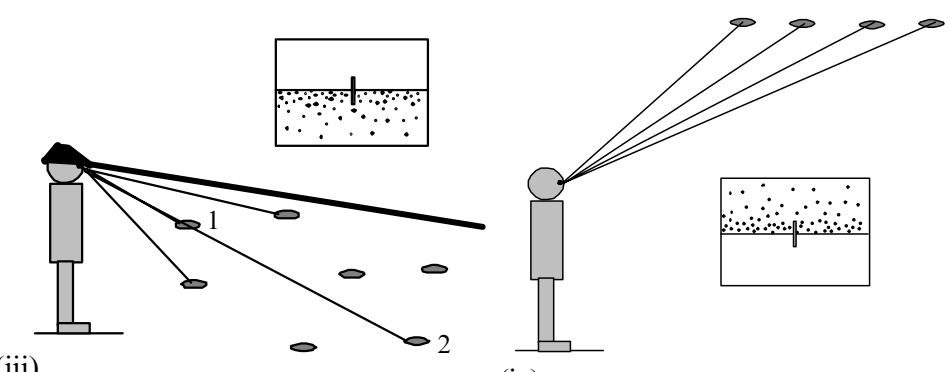

(iv)

Figure 1. The four display configurations were: (i) a standard cloud of dots with uniform 3-D dot density and dots extending well above and below eye-height; (ii) a standard ground plane with uniform density along the plane; (iii) a capped cloud; (iv) a ceiling (reflection of the ground plane about eye level). Insets show 2-D projection arrays.

Dots beyond $40 \mathrm{~m}$ from the viewpoint were culled from view in all except the capped-cloud condition. The capped cloud was a display with a 2-D layout (retinal locus and mean speed) identical to the ground plane, but without the height-in-scene depth order. To achieve this we took a standard cloud stimulus and applied the following manipulations: (a) increase dot density; (b) reduce the extent of the cloud below the observer's head to just a little $(\sim 1 \mathrm{~m})$ beneath the observer's feet; (c) extend the culling distance from $40 \mathrm{~m}$ by $\sim 10 \mathrm{~m}$; (d) mimic the vision of a flat-cap wearer with the peak of the cap tilted slightly downwards. This leaves a display in which the depth of a point in the capped-cloud condition is ambiguous. It can be seen in (iii) that points 1 and 2 will appear at the same height in the 2-D array. Note that a point at the horizon may be at any depth $(0.001 \mathrm{~m}$ to $50 \mathrm{~m})$ but a point towards the bottom of the display is restricted to a range of near distances $(0.001 \mathrm{~m}$ to $2.1 \mathrm{~m})$.

To control for the difference in motion distribution parameters we designed a new display, the 'capped cloud' (see figure 1). The capped cloud had an identical retinal motion distribution (locus and mean speed) to a ground plane, but without its height-in-scene depth order. Thus the capped-cloud condition allows us to tease apart whether performance differences between a ground plane and a 3-D cloud display are due to differences in retinal motion distribution or to differences in depth. In experiment 1 we explore the role of the retinal motion distribution and pictorial depth in the control of heading from flow.

\subsection{Binocular advantage - an issue of depth or correspondence?}

The second line of evidence in favour of the use of depth order in heading was that, when binocular disparity was added to a display, noise was less disruptive to the judgment of heading (van den Berg and Brenner 1994b). Again, a re-examination suggests that disparity could have improved performance without providing a specific depth cue. An alternative role for disparity could be in aiding the solution of the motion correspondence problem - the problem of matching dots between frames. Labelling elements with disparity allows a dot to be disambiguated from its neighbours in the Cyclopean array. Hence it is possible that the previously reported disparity advantage could be due to a simplification of the matching problem rather than depth ordering per se. 
In experiments 2 and 3 we explore the contribution of binocular disparity to the control of heading. In addition to the conditions used by van den Berg and Brenner (1994b) we add a display with nonveridical disparity, ie the disparity depth of a point is not consistent with its motion in the frontoparallel plane. This allows us to compare performance with veridical disparity (depth and correspondence information), nonveridical disparity (correspondence information only), and no disparity.

\section{Experimental design and rationale}

As noted, previous studies have explored the perception of linear heading and curvilinear paths by using psychophysical judgment tasks. In this paper we are concerned with a different issue: which properties of the visual flow field inform the active perceptual control of locomotor heading (steering). Therefore we chose to employ a task that closely matched the demands of natural steering with trials that lasted for $8 \mathrm{~s}$, to capture steering as the output of a perceptual-motor feedback loop.

These experiments were not intended to address the strategies that observers might use to determine heading from flow, and so we did not attempt to restrict the use of any potential strategy that could be employed in the natural world. However, a strategy that can be naturally used is to couple gaze and heading - to look where you want to go, and then bring heading direction round to coincide (close the gaze angle down to zero). Allowing use of this strategy was not desirable for two reasons. First, this paper is concerned with visual information and flow - therefore we wished to preclude the use of extraocular information [see Rushton et al (1998) on the central role of egocentric direction in control of heading on foot]. Second, in laboratory simulations, when gaze and heading are coupled, the observer can use two strategies based upon artifactual cues: maintaining the edge rate (number of elements passing the edge of the display) at the left and right edges of the screen, or centring the target in the middle of the display. Either of these strategies (which would not be available under natural conditions) will ensure the observer is heading directly towards the target. Therefore we chose to uncouple gaze and heading.

It has been reported that the natural preference of the visual system during steering is to keep the object that is guiding movement in central vision (Land and Lee 1994). The majority of heading judgment studies do not respect this. However, one paradigm that does map onto this observation is the film-making procedure of 'Dolly and Pan' (Cutting et al 1992). In this paradigm the display corresponds to an observer (or camera) fixating a target whilst translating to the left or right of it. This fulfills our two requirements to keep the reference object in central vision whilst dissociating gaze and heading. We used an active 'Dolly and Pan' task with simulated locomotion on an initial trajectory which was randomly to the left or right of a target tower, fixed in the environment. The observer's task was to control heading by steering using a joystick, so as to travel straight to the tower. Gaze was continuously computeradjusted to keep the tower centred on the projected display.

Some heading judgment tasks lock gaze rotation to a fixed near environmental feature (eg Warren and Hannon 1988), whereas some add a constant rotation of gaze (eg Royden et al 1992). The latter corresponds to visually tracking a target that is circling around the observer. The 'Dolly and Pan' task is more similar to the former, and the relatively more common of the two situations (Warren 1995).

Last, some studies use non-expanding dots and some use sparse environments with a ground plane and a small number of objects. Provided the dots are small, the lack of expansion should not significantly impair motion-in-depth or time-to-contact (see Gray and Regan 1998; Rushton and Wann 1999). Therefore, we decided to use the former so as to allow a more direct comparison with previous studies of heading judgment. 


\section{Conditions and predictions}

\subsection{Experiment 1: The role of retinal motion distribution and depth}

We created four conditions. (i) A cloud and (ii) a standard ground plane (balanced for total number of dots and mean 2-D speed) allowed comparison of our steering results with previous judgment experiments. (iii) A 'ceiling', which was a reflection of the ground plane about the horizontal axis (the same depth-order information as the ground plane, and also a surface, but much less likely to occur in the real world) which allowed us to examine upper/lower visual field differences. (iv) A capped cloud that had an identical retinal motion (mean speed and retinal locus) to a ground plane but lacked its depth-order information (see figure 1 for details). If retinal motion distribution is the critical factor affecting performance, we expect similar performance in the cappedcloud and ground-plane conditions. If depth is critical, we expect performance for the capped cloud to be poorer than for the ground plane.

\subsection{Experiment 2: The role of depth from disparity}

We used a modified Wheatstone stereoscope to allow the introduction of binoculardisparity depth-order information into a cloud and created three conditions: (i) dots with veridical disparity and veridical-disparity-defined motion-in-depth; (ii) dots with nonveridical disparity and nonveridical-disparity-defined motion-in-depth; (iii) dots with no disparity depth-order information and no disparity-defined motion-in-depth - a 'synoptic' display (van den Berg and Brenner 1994b). In condition (ii) the dots were assigned disparities and corresponding motion-in-depth trajectories at random (drawn from a distribution with the same ranges as for the veridical case). Therefore depth defined by disparity and depth defined by motion in the Cyclopean plane would only correspond by chance. In this study we have a strong test of the use of depth, the nonveridical stereo condition. If depth is used in perception of heading, the depth and heading systems can be described as coupled. Formal models of coupled systems (see Shallice 1988) indicate that coupled systems cannot take up contradictory states. ${ }^{(1)}$ Therefore, the critical nonveridical disparity condition should produce markedly poorer performance if depth is used. This approach stands in contrast to that used by van den Berg and Brenner (1994b). They compared a veridical disparity condition with a synaptic condition and manipulated the amount of noise. They found an interaction between noise and disparity, and concluded that "disparities improve judgments of heading".

The results they obtained are compatible with the particular model of heading that they advance, which involves an input for depth. However, their results are also compatible with other models consisting of independent systems for depth and heading, but non-independent noise.

In contrast, our nonveridical disparity manipulation predicts a clear result if the system for disparity depth is linked to that for heading.

If disparity depth order is used in the perceptual control of heading (steering) from flow, then performance should be elevated in condition (i) and impaired in condition (ii) relative to the nonstereo conditions. If disparity aids matching, then conditions (i) and (ii) should reveal a similar increase in performance.

\subsection{Experiment 3: Disparity and noise}

Experiment 2 should establish whether depth from disparity is used in steering. Experiment 3 was the same as experiment 2 but introduced a high level of noise (signal-tonoise ratio $=1$ ) to allow comparison of our results with van den Berg and Brenner's perceptual judgment studies.

(1) Consider a widely accepted example of coupled systems, or links between neural systemsthe link from vergence to accommodation: here we find that manipulation of the first system produces measurable changes in the second under natural viewing conditions. 


\section{General methods}

\subsection{Participants}

Observers with a range of experience at performing the heading task were deliberately chosen. The spread of relative observer performance in figures $2 c, 4 a$, and $4 b$ reflects the range of experience. With the exception of SKR (experiments 1-3) and JMH (experiments 2 and 3), observers were naive as to the experimental hypotheses.

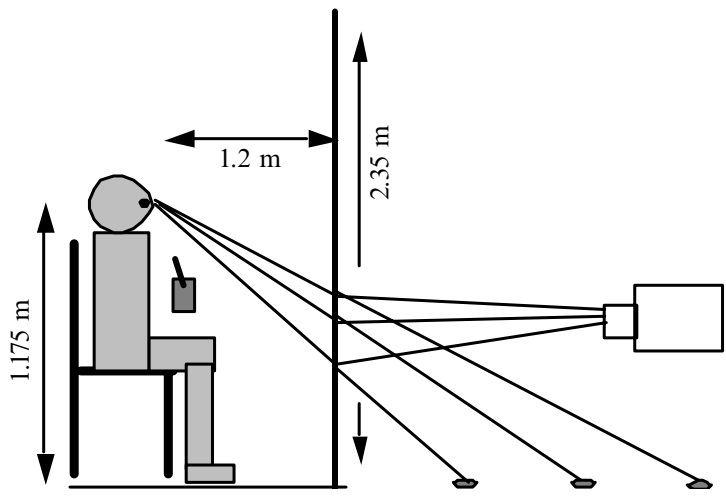

Figure 2. Back-projection screen $(80$ deg $\times 80 \mathrm{deg})$ with a resolution $1024 \times 768$ pixels. The analog joystick was fed through an 8-bit analog-to-digital converter. Latencies incurred in reading the device were less than $1 \mathrm{~ms}$ so allowing responses to be used in the rendering of the next frame. The joystick controlled the curvature of the heading trajectory (similar to a car) rather than the absolute heading angle.

\subsection{Apparatus and displays}

The display environments were built from randomly distributed dots with a constant number in view at any time (experiment 1: $400 \pm 10 \%$; experiments 2 and 3: $100 \pm 10 \%$ ). Each dot had a lifetime of less than $0.5 \mathrm{~s}$ (experiment 1: $400 \mathrm{~ms}$; experiments 2 and 3: $\sim 440 \mathrm{~ms}$ ). Initial direction of travel, at a speed of $2 \mathrm{~m} \mathrm{~s}^{-1}$ was to the left or right of a target tower set $20 \mathrm{~m}$ away (experiment $1: 10^{\circ} \pm 20 \%$; experiments 2 and $3: 15^{\circ} \pm 20 \%$ ). Temporal resolution was as high as could be reliably sustained (experiment 1: $30 \mathrm{~Hz}$; experiments 2 and 3: $18 \mathrm{~Hz}$ ). Displays were viewed in a darkened room. The display screen for experiment 1 was a back-projection screen $(80 \mathrm{deg} \times 80 \mathrm{deg})$ with a resolution $1024 \times 768$ pixels. For experiments 2 and 3 we used a CRT viewed through a Wheatstone stereoscope (horizontal field of view $20 \mathrm{deg}$; see figure 3). Display resolution was $481 \times 768$ pixels per eye with 2 -D sub-pixel addressing (32 calibrated linear grey levels) by the 'Backus method' (Georgeson et al 1996) to increase spatial resolution. Binocular disparities ranged from approximately 0 to $20 \mathrm{~min}$ of arc. The screen (or angular) velocities of individual dots varied depending on their distance from the observer, their eccentricity, and the course steered by the observer: a large heading error results in additional projected lateral motion and fast dot speeds. As an example, consider a dot positioned at a 20 deg eccentricity, $4 \mathrm{~m}$ from the observer, who is moving at $2 \mathrm{~m} \mathrm{~s}^{-1}$ (a very close and very eccentric point). Its velocity would be $23 \mathrm{deg} \mathrm{s}^{-1}$.

\subsection{Performance measures}

Heading error was defined as the difference between gaze (direction of target) and heading direction (tangent to path at each instance) (see figures 4 and 5). Heading error was recorded throughout the trial (see figures 3 and 4). There were 25 trials per condition and the conditions were randomly interleaved.

Statistical analysis and summary graphs use mean heading error over the full time course $(8 \mathrm{~s})$. Preliminary analysis of the group data revealed that the mean heading error was a useful measure. The relative performance remains approximately constant throughout the time course. In other words, if the ordering of conditions by heading error is $x>z>y$ at the beginning of the time course then it is also the ordering at the end of the time course. Further, although heading error at the end of the time course is found to correlate with heading error at the beginning of the time course, it does not constrain it. This was revealed by running some additional trials with two very different initial 

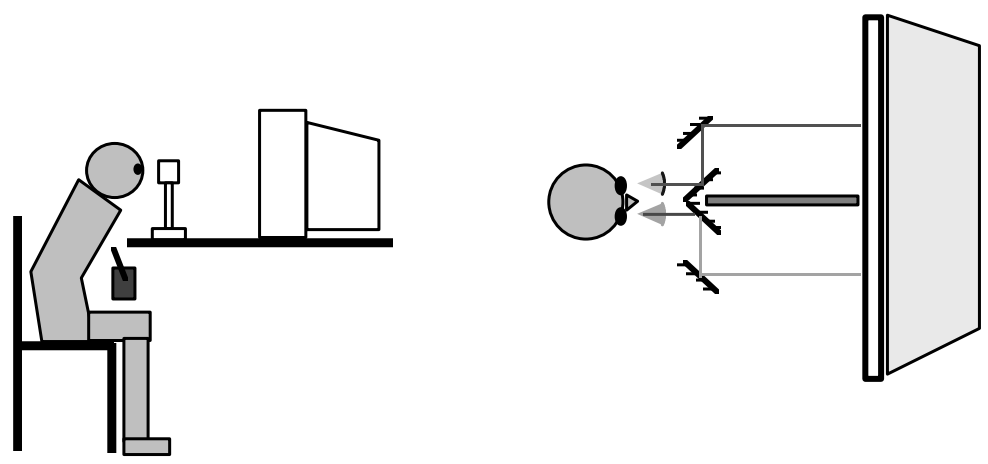

Figure 3. CRT viewed through a Wheatstone stereoscope (horizontal field of view $20 \mathrm{deg}$ ). Display resolution was $481 \times 768$ pixels per eye with 2 -D sub-pixel addressing (32 calibrated linear grey levels) using the 'Backus method' (Georgeson et al 1996) to increase spatial resolution.

Disparity was introduced into the displays by shifting the horizontal position of the dots in the left and right images. For each dot, the magnitude of the pixel shift was calculated from the distance from the observer of the frontoparallel plane that contained the dot [by using the smallangle approximation, Cormack and Fox (1985)]. In the nonveridical disparity condition the pixel shift was calculated by using the distance from another point in the scene, for instance the disparity of dot $x$ would be based upon the distance of dot $x-1$, the disparity of dot $x+1$ was based upon the distance of dot $x$. Dots were distributed randomly in space so there was no correlation between the disparity of a dot and its distance (and hence motion in the frontoparallel plane of the cyclopean array). This procedure ensured that the distribution of disparity depths was identical in the veridical and nonveridical conditions. Also, it produced coherent motion-in-depth trajectories for the dots in both conditions.

In experiment 3, noise was introduced by a similar technique to that previously employed by van den Berg and Brenner (1994b). The 2-D velocity for each point was determined and a noise component calculated and added that had the same magnitude but a random direction. This produced a signal-to-noise ratio of 1 which was the highest employed previously (van den Berg and Brenner 1994b). The same noise was added in all conditions, and in the stereo cases the noise was always of the same sign for left and right eyes, so producing a perturbation in the $x y$-plane rather than in $z$-depth.

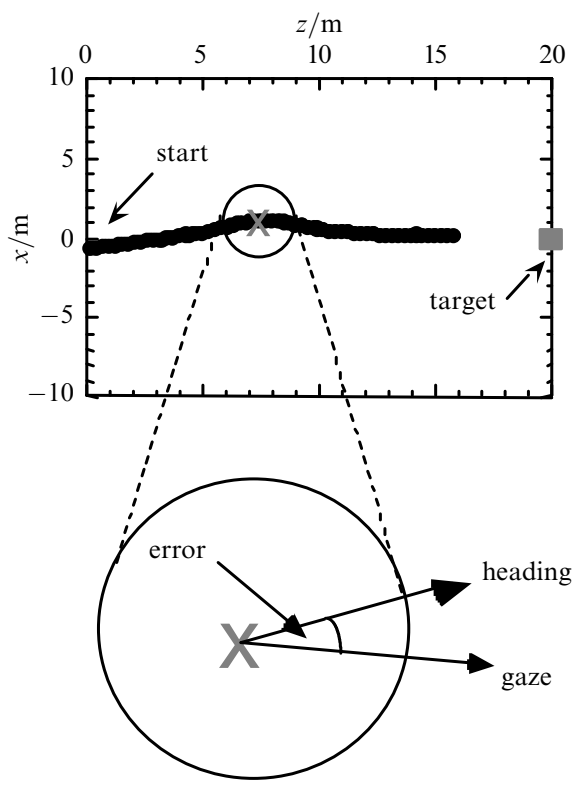

Figure 4. An example trial showing observer's progress towards the target tower (top panel). The circle shows an area that has been enlarged below to indicate the angles we refer to. Heading error was defined as the difference between gaze (direction of target) and heading direction (tangent to path at each instant). 


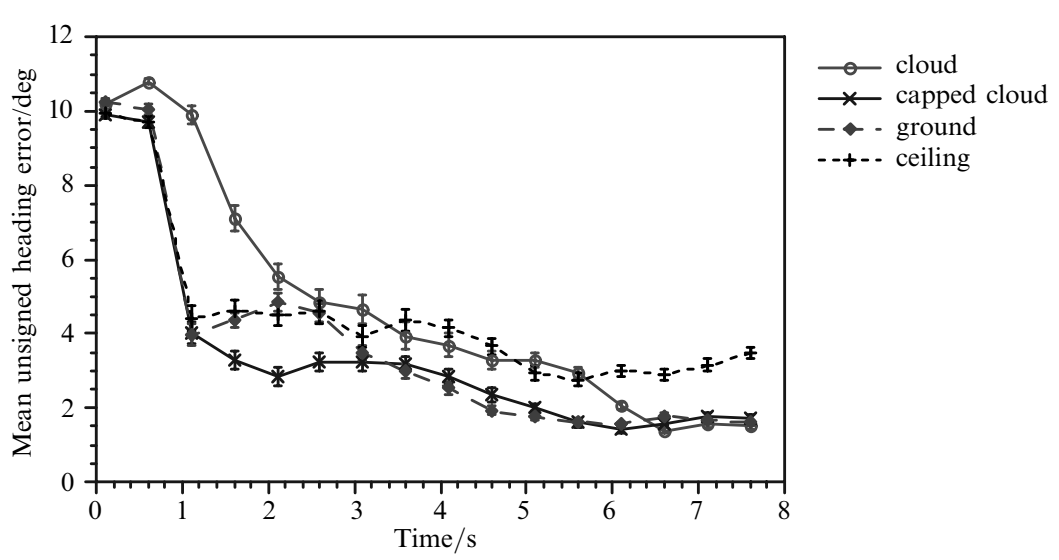

Figure 5. An example plot of results for observer VJ. Mean unsigned heading error was sampled with $200 \mathrm{~ms}$ windows at $0.5 \mathrm{~s}$ intervals across the time course. Note how error is systematically reduced from an initial $10 \mathrm{deg}$. Mean errors across the whole trial for the above observer are: $4.632 \mathrm{deg}$ (cloud), $3.250 \mathrm{deg}$ (capped cloud), $3.507 \mathrm{deg}$ (ground), $4.377 \mathrm{deg}$ (ceiling). Vertical bars indicate standard error.

heading errors (10 deg to the left or right of the target versus $20 \mathrm{deg}$ ). It was found that by the midpoint of the time course the observer had brought the heading error down to approximately the same value in both conditions. Performance then remained approximately equal throughout the remainder of the trial. Care should be taken in attempting to compare these results directly with those from previous judgment tasks; see example time-course plot in figure 5.

\section{Results and discussion}

\subsection{Experiment 1}

Heading error was recorded for the cloud, ground, capped-cloud, and ceiling conditions. An analysis of variance showed a significant main effect of condition $(p<0.0001)$. Pairwise comparisons revealed that the cloud condition produced significantly worse performance than the other conditions (ground plane, $p<0.0001$; capped cloud, $p<0.0001$; ceiling, $p<0.0001)$. There was no significant difference in performance between the ground plane and capped cloud $\left(F_{124}=0.062\right.$, ns). The ceiling condition produced a level of performance intermediate between the cloud, and the ground $(p<0.0001)$ and capped cloud $(p<0.0001)$, demonstrating an interesting lower visual field advantage [see Previc (1990) and Rubin et al (1996) for other visual hemifield differences] (see figure 6). These results suggest that the difference in 2-D layout rather than 3-D layout is responsible for the decrement in performance observed with cloud displays. This finding questions the use of pictorial depth in steering from flow.

\subsection{Experiment 2: Does binocular disparity aid steering?}

Data were collected and analysed as for the first experiment. The three conditions used were veridical disparity, nonveridical disparity, and no disparity. No difference was found between conditions $\left(F_{2,240}=0.085\right.$, ns) and pairwise comparisons revealed no simple effects (see figure 7a). Performance levels in the comparable cloud condition were similar to those in the previous 3-D layout experiment. The lack of elevation of performance suggests that disparity is not used in steering. Failure to find a clear benefit for veridical disparity is in fact in line with that previously reported in a heading judgment task (van den Berg and Brenner 1994b). Importantly, as performance was not impaired when depth and heading were not congruent (nonveridical stereo), we are led to conclude that the perceptual systems associated with depth and the control of heading (steering) from flow are not coupled. 


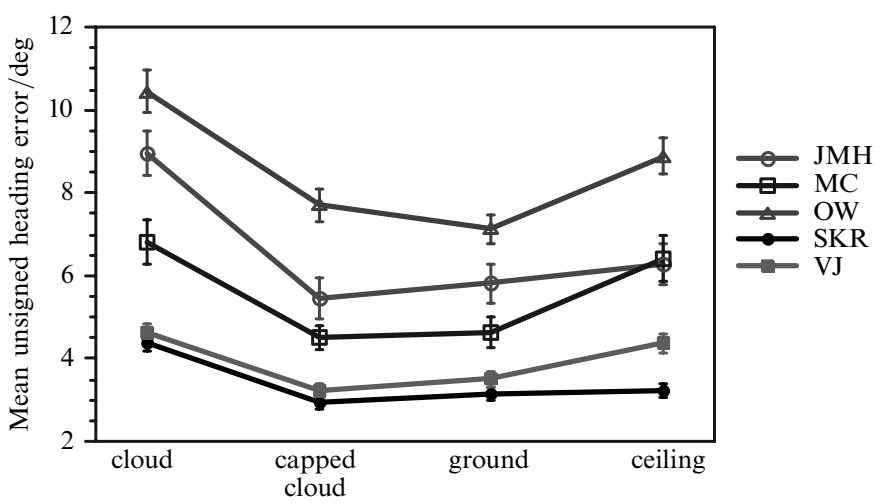

Figure 6. Interaction plot (condition and observer) of mean unsigned heading error over time course with standard errors indicated by vertical bars.

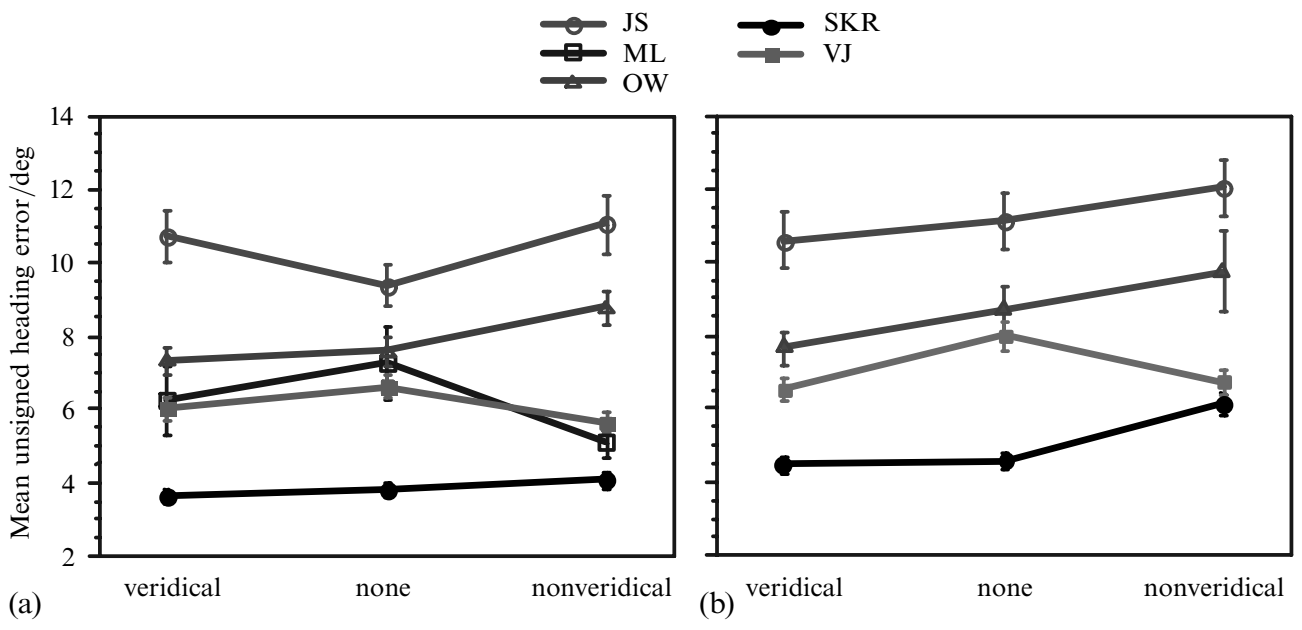

Figure 7. (a) No noise, interaction plot (condition and observer) of mean unsigned heading error over time course. (b) Noise (signal-to-noise ratio $=1$ ), interaction plot (condition and observer) of mean unsigned heading error over time course. Standard errors are indicated by vertical bars.

\subsection{Experiment 3: Disparity and steering in a noisy stimulus}

The aim of this experiment was to determine if steering and heading judgments demonstrate a comparable pattern of results. Following van den Berg and Brenner (1994b) we tried to open up a performance difference between the three disparity conditions through the addition of a high level of noise. When a very high level of noise (signalto-noise ratio $=1$ ) was added, the main effect of condition almost reached significance $\left(F_{2,192}=2.965, \mathrm{~ns}\right)$ (see figure $\left.7 \mathrm{~b}\right)$. Pairwise comparisons revealed that veridical disparity was not significantly better than the no-disparity condition $\left(F_{124}=1.953\right.$, ns $)$, nor did the difference between nonveridical disparity and the no-disparity conditions reach significance $\left(F_{124}=1.058\right.$, ns). However, a significant difference was found between veridical disparity and nonveridical disparity conditions $\left(F_{124}=5.885, p<0.05\right)$ (see figure 4). These results are similar to those reported previously on heading judgments, but, as discussed in section 1, our interpretation is somewhat different. 


\section{Discussion}

Here we tackle a few potential concerns about the experimental design, then discuss the implications of our results.

\subsection{Depth gradient in capped cloud}

Performance in the capped-cloud condition was as good as for a ground plane, suggesting that 2-D layout, rather than 3-D depth, is responsible for performance differences in previous studies. There are cues to depth, however, that do arise for the capped cloud. The depth range in the cloud of a point towards the bottom of the screen is more limited than the depth range of a point towards the top of the screen (see caption for figure 1). Hence the mean, median, modal, or maximum distance of a point increases from the bottom to the top of the display, and this could serve as a probabilistic cue to depth. Further, points at the bottom of the screen are always near (see figure 1). In principle, an observer could deduce this and then concentrate on the movement of these points. They are potentially informative because their vectors are least contaminated by an additional rotational component due to gaze movement. However, in our displays there were very few of these points and they moved very fast.

Let us consider the specific hypothesis proposed to account for heading judgment results. Decomposition of the retinal flow field by rotation subtraction uses the movement of distant objects (van den Berg 1992). In the capped cloud, there is no part of the projected array that contains just distant objects. To assume that points near the top of the screen are distant would lead to errors and is the same as assuming that points at the same image position in a cloud are distant. Therefore these steering results appear incompatible with the rotation subtraction hypothesis.

An additional point to consider is the upper/lower visual field effect (ceiling versus ground plane). A ceiling and a ground plane contain identical depth-order cues and are also both surfaces. The relative performance differences support the theory that retinal locus is important in the perception and control of heading.

\subsection{Rotation rates}

A large number of recent studies have been concerned with rotation rates. Could it be that our result does not hold at a 'higher rotation rate' (ie as would occur if the foot speed or initial heading error were increased)? This question illustrates the problems with taking psychophysical judgment tasks as a model of natural control of steering. First, in a steering context the rotation rate that observers are exposed to depends upon their responses. If they do not attempt to correct their heading direction then the rotation rate will increase. In an active control context, however, high rotation rates may actually be considered as information. If whilst fixating the target observers note a large amount of rotational flow, then they know that they are not heading towards it. If changing the direction of travel reduces the rotational flow, then they have just turned towards the target. Rather than attempt to determine the instantaneous direction of travel and the instantaneous direction of the target and compare them before modifying the direction of travel, observers can instead simply use an iterative solution whereby they turn in the direction that reduces the rotational component.

Second, we question whether the high rotation rates that are introduced in psychophysical judgment tasks are valid for natural contexts and actions. Gaze rotation rate is a function of the instantaneous angle, $\alpha$, of the fixation point from the locomotor trajectory, the distance $Z$ of the observer from where a linear trajectory will pass nearest to the fixation point, and the locomotor speed, $V$. For locomotion at a constant velocity we can consider the instantaneous time before the observer passes the fixation point as $T_{\mathrm{p}}=Z / V$. The gaze rotation rate, $w$, is then specified as:

$$
w=0.5 \sin (2 \alpha) V / Z=0.5 \sin (2 \alpha) / T_{\mathrm{p}} .
$$


This equation illustrates that, to achieve high rotation rates, the fixation point must either be eccentric or the time to passage relatively short (in other words, the distance in depth between observer and fixated point should be short). Hence, a rotation rate of $5 \mathrm{deg} \mathrm{s}^{-1}\left(0.0873 \mathrm{rad} \mathrm{s}^{-1}\right)$ will be achieved for a fixation point offset from the instantaneous locomotor trajectory by $\alpha=5 \mathrm{deg}$, but only $1 \mathrm{~s}$ before it is passed. For a fixation point that has moved by $\alpha=20 \mathrm{deg}$ from the locomotor trajectory, the rotation rate will rise to $5 \mathrm{deg} \mathrm{s}^{-1}$ at a $T_{\mathrm{p}}=3.68 \mathrm{~s}$, but within $1 \mathrm{~s}$ the trajectory will have taken the observer $28 \mathrm{deg}$ eccentric to the target, and within $2 \mathrm{~s}$ the fixation point will be at $\alpha=44 \mathrm{deg}$ eccentricity. These eccentricities are at the limits of conventional displays and probably beyond normal practice. If a car driver maintained fixation on a pedestrian for $2 \mathrm{~s}$ and until his/her gaze was at $45 \mathrm{deg}$ to the road, it would not be surprising to note that his/her heading perception was poor. In natural settings such rotation rates are only encountered transiently and the human gaze system is well suited to using alternating fixations to avoid the kind of sustained rotation rates used in previous psychophysical settings.

\subsection{Heading judgments versus control of heading}

The data presented here demonstrate the same pattern as those of van den Berg and Brenner (1994b) for perceptual judgments, that, when a large amount of noise is added, performance is better with depth than without it. However, we argue that under natural conditions, when steering around an environment, disparity depth has no influence on performance. Our interpretation of the results when noise is added is different from the previous study (see section 1).

Why did we find a small performance difference with a very high level of noise? Neural systems for heading and depth inevitably share a commonality of information or anatomical inputs and outputs; therefore it is unlikely that the systems for depth and heading are absolutely isolated. For instance, it is likely that both are implicated in the perception of environmental layout. If so, contradictory outputs of the two systems will increase a general level of uncertainty, consistent ones will reduce it, and so impact on performance. Thus, a performance difference found only in the presence of large amounts of noise is not enough to suggest that depth is used to aid heading under normal viewing conditions.

\section{Summary}

Although depth may be used in other aspects of locomotor control, here we find a functional independence of the perceptual systems for depth and the control of heading from flow. The presence of depth information (either height-in-scene or disparity) does not confer advantage for the perceptual control of heading (steering). Whatever strategy is used to steer using optic flow, it is not aided by providing either height-in-scene or disparity depth. Retinal motion distribution, not depth, is the major determinant of performance for the perceptual control of heading from flow.

Acknowledgements. We wish to thank David Lee, Albert van den Berg, Simon Watt, and anonymous referees for providing useful comments on an earlier version of this manuscript. This work was supported by an EPRSC grant to SKR and JPW and an MRC grant to JMH.

\section{References}

Albright T D, 1984 "Direction and orientation selectivity of neurons in visual area MT of the macaque" Journal of Neurophysiology 521106 - 1130

Berg A V van den, 1992 "Robustness of perception of heading from optic flow" Vision Research $321285-1296$

Berg A V van den, Brenner E, 1994a "Humans combine the optic flow with static depth cues for robust perception of heading" Vision Research $342153-2167$

Berg A V van den, Brenner E, 1994b "Why two eyes are better than one for judgements of heading" Nature (London) $371700-702$ 
Cormack R, Fox R, 1985 “The computation of retinal disparity” Perception \& Psychophysics 37 $176-178$

Crowell J A, Banks M S, 1993 "Perceiving heading with different retinal regions and types of optic flow" Perception \& Psychophysics 53 325-337

Crowell J A, Banks M S, 1996 "Ideal observer for heading judgements" Vision Research 36 $471-490$

Cutting J E, 1996 "Wayfinding from multiple sources of local information in retinal flow" Journal of Experimental Psychology: Human Perception and Performance 22 1299-1313

Cutting J E, Springer K, Baren P A, Johnson S H, 1992 "Wayfinding on foot from information in retinal, not optical, flow" Journal of Experimental Psychology: General 102 41-72, 129

Ehrlich S M, Beck D M, Crowell J A, Freeman T C A, Banks M S, 1998 "Depth information and perceived self-motion during simulated gaze rotations" Vision Research 38 3129-3145

Georgeson M A, Freeman T C A, Scott-Samuel N E, 1996 "Sub-pixel accuracy-psychophysical validation of an algorithm for fine positioning and movement of dots on visual-displays" Vision Research $36605-612$

Gibson J J, 1950 The Perception of the Visual World (Westport, CT: Greenwood)

Gibson J J, 1979 The Ecological Approach to Visual Perception (Hillsdale, NJ: Lawrence Erlbaum Associates)

Goodale M A, Milner A D, 1992 "Separate visual pathways for perception and action" Trends in Neuroscience $1520-25$

Gray R, Regan D, 1998 "The relevance of heading and motion-in-depth data collected with constant-sized dot displays" Investigative Ophthalmology \& Visual Science 39(4) S982

He Z J J, Nakayama K, 1994 "Perceived surface shape not features determines correspondence strength in apparent motion" Nature (London) $367173-175$

Land M F, Lee D N, 1994 "Where we look when we steer" Nature (London) $369742-744$

McKee S P, Nakayama K, 1984 "The detection of motion in the peripheral visual field" Vision Research $24491-500$

Milner A D, Goodale M A, 1996 The Visual Brain in Action (Oxford: Oxford University Press)

Palmisano S, 1996 "Perceiving self-motion in-depth - the role of stereoscopic motion and changingsize cues" Perception \& Psychophysics $581168-1176$

Previc F H, 1990 "Functional specialization in the lower and upper visual fields in humansits ecological origins and neurophysiological implications" Behavioural and Brain Sciences $\mathbf{1 3}$ $519-575$

Regan D, Beverley K I, 1982 "How do we avoid confounding the direction we are looking and the direction we are moving?" Science 215 194-196

Roy J P, Wurtz R H, 1990 "The role of disparity-sensitive cortical-neurones in signalling the direction of self-motion" Nature (London) 348 160-162

Royden C S, Banks M S, Crowell J A, 1992 "The perception of heading during eye movements" Nature (London) $\mathbf{3 6 0} 583-585$

Rubin N, Nakayama K, Shapley R, 1996 "Enhanced perception of illusory contours in the lower versus upper visual hemifields" Science $271651-653$

Rushton S K, Harris J M, Lloyd M R, Wann J P, 1998 "Guidance of locomotion on foot uses perceived target location rather than optic flow" Current Biology 8 1191-1194

Rushton S K, Wann J P, 1999 "Weighted combination of size and disparity: A computational model for timing a ball-catch" Nature Neuroscience 2 186-190

Sedgwick H, 1986 "Space perception", in Handbook of Perception and Human Performance volume 1, Eds K R Boff, L Kaufman, J P Thomas (New York: John Wiley)

Shallice T, 1988 From Neuropsychology to Mental Structure (Cambridge: Cambridge University Press)

Stone L S, Perrone J A, 1997 "Human heading estimation during visually simulated curvilinear motion" Vision Research $37573-590$

Turrano K, Wang X, 1994 "Visual discrimination between a curved and straight path of self motion: effects of forward speed" Vision Research $34107-114$

Warren W H, 1995 "Self-motion: visual perception and visual control", in Perception of Space and Motion Eds W Epstein, S Rogers (San Diego, CA: Academic Press) pp 263-312

Warren W H, Hannon D J, 1988 "Direction of self-motion is perceived from optic flow" Nature (London) $336162-163$ 